Anales de Geografía de la Universidad Complutense ISSN: 0211-9803

http://dx.doi.org/10.5209/AGUC. 62481

\title{
La diferenciación residencial reciente en el municipio de Málaga. Una aplicación del Î́ndice de Theil
}

\author{
Ana Ester Batista Zamora ${ }^{1}$; Juan José Natera Rivas ${ }^{2}$ \\ Recibido: 10 de julio del 2017/ Enviado a evaluar: 21 de julio del 2017/ Aceptado: 18 de octubre del 2018
}

Resumen. El municipio de Málaga ha venido experimentado pérdidas sistemáticas de población extranjera desde 2013, revirtiendo la tendencia que exhibía desde comienzos de siglo, y podemos preguntarnos qué impacto han tenido estas pérdidas sobre los niveles de diferenciación residencial de los extranjeros. Para ahondar en la cuestión emplearemos el Índice de Theil, que además de indicar los niveles de diferenciación, permite identificar los aportes que la segregación entre grupos (españoles/extranjeros, extranjeros entre sí) realiza a los niveles de segregación total. Utilizando como unidad espacial las macroáreas municipales, la aplicación del índice indica que los niveles de diferenciación residencial, bajos de por sí, han disminuido en estos tres años, al tiempo que han quedado registradas no sólo la influencia que sobre la composición interna de la diferenciación han tenido la construcción de nuevos complejos residenciales, sino la existencia de macroáreas donde el principal monto de la diferenciación se debe a las diferencias residenciales entre extranjeros.

Palabras clave: Diferenciación residencial multigrupo; Índice de Theil; Inmigrantes extranjeros.

\section{[en]. Recent residential differentiation in the municipality of Malaga. An application of Theil Index}

\begin{abstract}
The ammount of foreigners living at the municipality of Malaga has been shrinking since 2013, reversing the trend they had shown since the beginning of the century, and we can ask about the impact these losses have had on the foreigners's levels of residential differentiation. To get into this issue, we will use the Theil Index, which not only indicates the levels of residential differentiation, but also allows us to identify the extent that segregation between groups (Spanish/foreigners, foreigners/foreigners) contibutes to the levels of total segregation. Using the municipal macroareas as a spatial unit, the application of the index indicates that levels of residential differentiation, albeit low, have declined in these three years. Along with that, the index has registered the influence of the erection of new residential complexes, and the existence of macro areas where the main amount of differentiation is due to residential differences between foreigners.
\end{abstract}

Keywords: Multigroup residental differentiation; Theil Index; Foreign inmigrants.

1 Departamento de Geografía. Universidad de Málaga.

E-mail: anabatista@uma.es

2 Departamento de Geografía. Universidad de Málaga.

E-mail: jjnatera@uma.es 


\title{
[fr] Différenciation résidentielle récente dans la ville de Malaga. Une application de 1'Indice Theil
}

\begin{abstract}
Résumé. Le nombre d'étrangers vivant dans la municipalité de Malaga a diminué depuis 2013, inversant la tendance qu'ils avaient manifestée depuis le début du siècle, et nous pouvons nous interroger sur l'impact de ces pertes sur les niveaux de différenciation résidentielle des étrangers. Pour analyser ce problème, nous allons utiliser Theil Index, qui indique non seulement les niveaux de différenciation résidentielle, mais nous permet également d'identifier dans quelle mesure la ségrégation entre les groupes (espagnols / étrangers, étrangers / étrangers) s'élève aux niveaux de total ségrégation. En utilisant les macroéconomies municipales en tant qu'unité spatiale, l'application de l'indice indique que les niveaux de différenciation résidentielle, quoique bas, ont diminué au cours de ces trois années. Parallèlement, l'indice a enregistré l'influence de l'érection de nouveaux complexes résidentiels et l'existence de macrodomaines où la principale différence de différentiation est due aux différences de résidence entre les étrangers.
\end{abstract}

Mots clés: Multigroup différenciation résidentielle; Indice Theil; Immigrants étrangers.

Cómo citar. Batista Zamora, A.E. y Natera Rivas, J.J. (2018): La diferenciación residencial reciente en el municipio de Málaga. Una aplicación del Índice de Theil. Anales de Geografía de la Universidad Complutense, 38(2), 289-311.

Sumario. 1. Introducción. 2. Sobre la segregación residencial. 3. Fuentes y metodología. 4. Los niveles de segregación en el municipio de Málaga. 4.1. La segregación multigrupo. 4.2. La segregación intergrupo. 5. Conclusiones. 6. Bibliografía.

\section{Introducción}

En 2016, última referencia temporal disponible en el momento de escribir estas líneas, había un total de 42.916 empadronados de nacionalidad extranjera en el municipio de Málaga, un 7,54\% del total de la población empadronada ese año. Esta cifra 13 años antes (2003 es el primer año para el que contamos con información padronal a nivel de barrio) era de tan sólo 17.920 personas, y fue 2013 el año en el que mayor cantidad de extranjeros residían en el municipio, 50.394 personas, el 8,86\% del total; una cifra que, desde entonces, ha venido menguando año a año hasta llegar a la actual.

La motivación para residir en Málaga por parte de esta población es mayoritariamente laboral : marroquíes, rumanos, ucranianos, nigerianos y paraguayos son, por ese orden, las principales nacionalidades presentes en 2016, sumando entre todas ellas 21.307 efectivos, la mitad de los extranjeros empadronados; por su parte, las nacionalidades cuyas motivaciones son más residenciales que laborales, aunque presentes, están muy por debajo de ellos -alemanes y británicos, por ejemplo, no alcanzan los 750 individuos, y son la decimoctava y decimonovena nacionalidad, respectivamente.

La distribución espacial de los extranjeros en el municipio de Málaga nunca fue homogénea: tomados como conjunto, se concentran básicamente en el área pericentral y en las expansiones históricas al oeste del mismo, en tejido levantado a partir de los 
años sesenta. Sobre este panorama general, discriminados por origen su distribución espacial varía: alemanes y británicos se concentran básicamente en los barrios del este malagueño, área con comparativamente buenas condiciones residenciales (en ella el precio de la vivienda es uno de los más elevado del municipio, 2.200 euros $/ \mathrm{m} 2$ en enero de 2016) y que en el imaginario colectivo continúa siendo una de las mejores para residir; los marroquíes lo hacen inmediatamente al norte y noroeste del centro histórico y en Palma/Palmilla, zona degradada que es también el principal asiento de los nigerianos. Por su parte, las mayores concentraciones de latinoamericanos están en Carretera de Cádiz y Cruz del Humilladero, esto es, en la expansión que conoció la ciudad durante los años sesenta y setenta en dirección sudoeste desde el centro, mientras que los rumanos, además de en Palma/Palmilla, se localizan en el litoral este, pero en uno de los barrios de peor calidad constructiva de la zona, Playas del Palo, que fue catalogado en su momento como "zona especialmente desfavorecida" (Egea et al., 2008).

Desde el punto de vista geográfico contamos con investigaciones que dan cuenta de la diversidad de orígenes de la población extranjera en la región metropolitana malagueña (Galeano y Bayona, 2016); otras, ya centradas en el municipio de Málaga, han tratado de comprobar el impacto que la llegada de extranjeros ha tenido sobre los volúmenes de población y la estructura por edad (Ocaña, 2005b; Natera y Batista, 2012), y sobre sus niveles de diferenciación residencial (Natera, 2012, Fernández, s.f.). En estas últimas el instrumento estadístico seleccionado es el Índice de Segregación, empleando como unidades espaciales de referencia el barrio y sección censal, respectivamente, siguiendo la tónica de la mayor parte de las investigaciones sobre segregación referidas a la realidad española, a los que Natera (2012) añade técnicas de autocorrelación espacial para comprobar la no aleatoriedad de las distribuciones espaciales e identificar agrupaciones estadísticamente significativas de extranjeros en el espacio urbano malagueño.

Sin embargo, aún no contamos con investigaciones que analicen el impacto que el reciente y notable desplome del número de extranjeros ha tenido sobre los niveles de diferenciación residencial del municipio. Las páginas que siguen tratan de llenar este hueco, comparando los niveles de diferenciación residencial de la población empadronada en el municipio de Málaga, diferenciada por su nacionalidad, correspondientes a 2013 y 2016. Sin embargo, el uso del instrumento que emplearemos, el índice de Theil, no está demasiado extendido en este tipo de estudio, lo que añade un valor añadido al que ahora presentamos. Un valor añadido que se deriva no tanto del empleo del índice, como de la posibilidad que nos brinda a la hora de descubrir si es la separación entre españoles y extranjeros, o la separación entre los seis grupos en los que hemos catalogado a los extranjeros, la que más aporta a los niveles de segregación totales, indicando, además, su evolución temporal reciente. 


\section{Sobre la segregación residencial}

La segregación residencial puede definirse, de manera simple, como la aglomeración geográfica de individuos que comparten una característica que se emplea para diferenciarlos del resto de la población urbana (Rodríguez, 2001). La localización de dichos individuos en el espacio urbano por lo general se realiza a partir de su lugar de residencia -de ahí el término "residencial"-, por ser éste el criterio empleado en los Censos y Padrones de población para georeferenciar a la población, las fuentes más utilizadas en este tipo de estudio. Y en cuanto a la característica que identifica a los componentes de un grupo, y que los diferencia de los demás, puede ser relativa a la estratificación socioeconómica (nivel de ingreso o de estudios, por ejemplo), o a la segmentación biosociocultural (raza, religión, nacionalidad, etc.). La variable que emplearemos en nuestro estudio pertenece a este último grupo, y es la nacionalidad de los empadronados en el municipio de Málaga.

Siendo la segregación residencial un hecho extremadamente común en nuestras ciudades, lo que diferencia sus manifestaciones son las bases a partir de las cuales surge, la profundidad de la misma, y las pautas espaciales resultantes. Sin olvidar la influencia que ejerce la estructura por edad y la evolución del ciclo familiar, en el caso de la población extranjera de las ciudades españolas la segregación residencial tiene una base fundamentalmente económica, puesto que es la capacidad de compra, la fuerza en el mercado inmobiliario, la que determina en mayor medida la calidad de la vivienda y el tipo de espacio urbano en el que tenderán a concentrarse los diferentes grupos de población. De cualquier forma, la profundidad de la segregación no es muy grande, puesto que, por un lado, los niveles de segregación son relativamente reducidos, y por lo general en descenso; y por otro, no se han detectado la formación de ghettos ni en ciudades (caso de Madrid -Echezarra, 2010- o Sevilla Huete y Muñoz, 2011) ni afectando a nacionalidades concretas (caso de los marroquíes -Achebak y Bayona, 2015). Tan sólo los europeos retirados presentarían niveles elevados de segregación, tal y como se desprendería de los estudios del levante español (Cutillas, 2009; Mantecón et al., 2009; Huete y Mantecón, 2011). En cuanto a las pautas espaciales, hay una clara división en la localización espacial de los extranjeros según que su motivación para residir entre nosotros sea laboral o residencial. Los primeros tienden a localizarse allí donde la vivienda es más asequible, vale decir en las áreas menos valoradas del tejido urbano, mientras que los residenciales se localizan en el extremo opuesto de la escala, en las zonas con mejores condiciones ambientales, o socialmente más valoradas, de nuestras ciudades.

En este sentido, ni los censos de población ni los padrones municipales de habitantes dan información sobre ingresos, pero la nacionalidad, que sí es recogida en ellos, suele emplearse como una proxi de los mismos: se ha indicado (Bonillo et al., 2011) que diferenciar a la población según su nacionalidad es, a grandes rasgos, equivalente a diferenciarla por sus ingresos potenciales; a modo de ejemplo, la renta de los extranjeros de la UE es sustancialmente superior a los extranjeros procedentes del resto del mundo, según los indicadores de calidad de vida del INE, mientras que, en el caso concreto de Andalucía, son los inmigrantes de países desarrollados y los 
latinoamericanos los que tienen los mejores trabajos, y los africanos, los peores (Martínez, 2011). En consecuencia, también es diferente su capacidad de elección de vivienda, su fuerza en el mercado inmobiliario.

\section{Fuentes y metodología}

La información estadística necesaria para realizar esta investigación ha sido obtenida de los padrones municipales de 2013 y 2016 -último disponible-, cuyos registros a nivel individual, referidos tanto a las secciones censales como a los barrios, pueden ser obtenidos en el Portal de Datos Abiertos del Municipio de Málaga . La población incluida en las dos bases de datos ha sido dividida en siete grupos, que se corresponden con agrupaciones ampliamente utilizadas en estudios sobre inmigración: españoles, latinoamericanos, magrebíes, subsaharianos, europeos orientales, Países Desarrollados, y resto de nacionalidades.

En cuanto a la división espacial, hemos optado por utilizar el barrio, agregándolos a nivel de macroáreas. Éstas son 19 divisiones espaciales empleadas por el Observatorio de Medioambiente Urbano (OMAU) para la realización de estudios a nivel intraurbano, y cuya delimitación (Figura 1) parte de la correspondiente a las 12 áreas histórico tipológicas identificadas en el planeamiento. Estas macroáreas están compuestas por un número variable de barrios, cuyos límites fueron establecidos en su momento por el Centro Municipal de Informática (CEMI) del Ayuntamiento malagueño. Estos barrios no son una mera adición de secciones censales -es más, la mayor parte de ellos "cabalgan" sobre porciones de varias secciones, y para su delimitación se emplearon diferentes criterios: delimitación contenida en los proyectos de urbanización, tipología de las viviendas, límites históricos ampliamente aceptados, usos del suelo - diferenciando usos industriales o grandes equipamientos de los usos residenciales, por ejemplo-, etc. El hecho de que el barrio sea la unidad espacial básica a partir de la que se construyen las unidades mayores tiene, al menos, dos ventajas. La primera es que el barrio es una unidad con sentido sociológico, mucho más que el que pueda tener la sección censal, $\mathrm{y}$, dados los criterios empleados para su delimitación, es homogéneo en sus características constructivas, tipológicas, etc. La otra ventaja es que, a diferencia de las secciones censales, la delimitación de los primeros no varía, lo que permite mantener invariada la unidad espacial, alejando a nuestro trabajo de la influencia que sobre el cálculo de los índices tiene la modificación de las unidades espaciales a las que la información estadística queda referida, el conocido problema de la Unidad Espacial Modificable (MAUP, por sus siglas en inglés). Por tanto, las variaciones de los valores del instrumento estadístico que hemos empleado no se derivan en proporción alguna de cambios en las unidades espaciales. 
Figura 1. Macroáreas del municipio de Málaga.

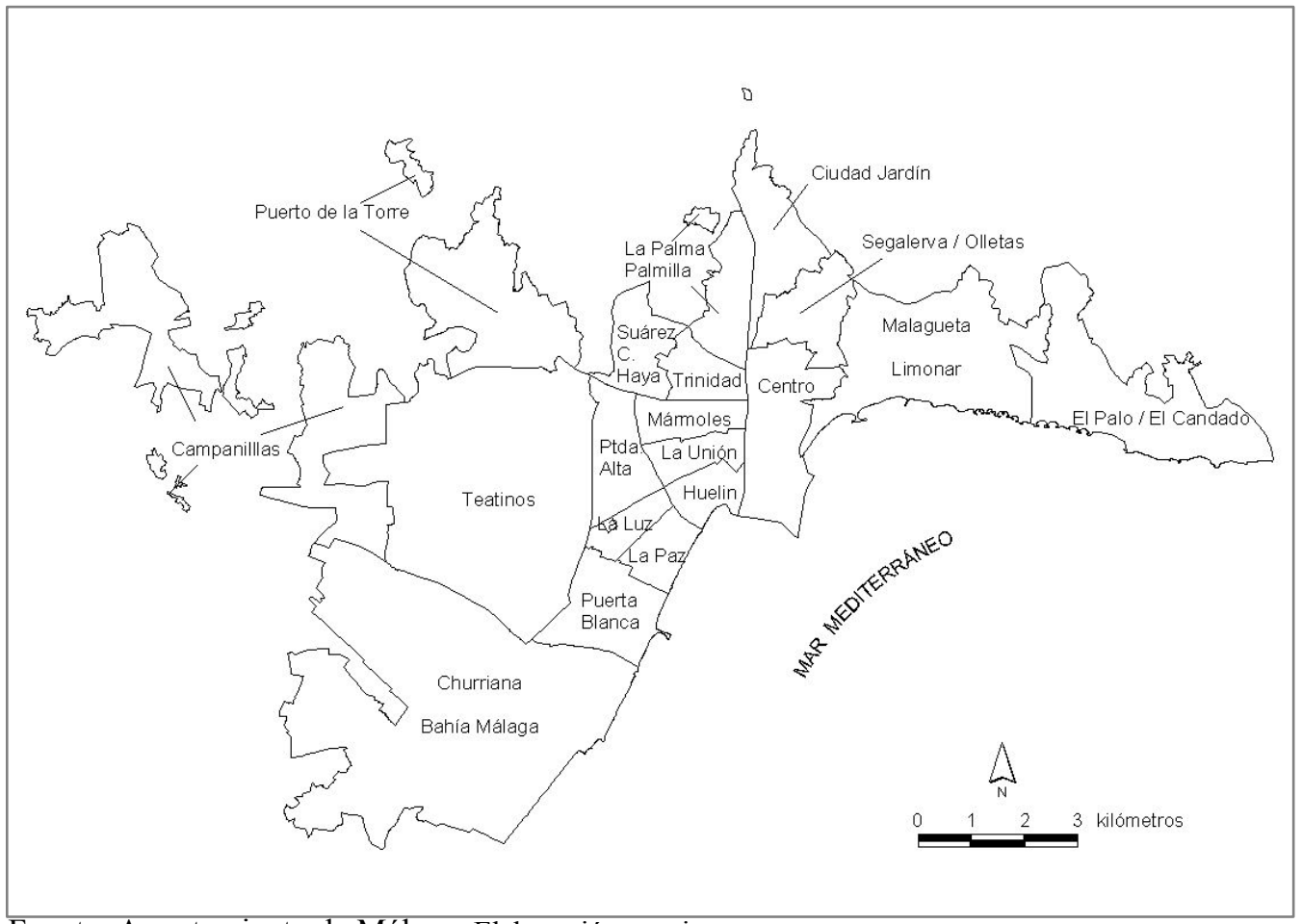

Fuente: Ayuntamiento de Málaga. Elaboración propia.

Independientemente de la unidad espacial empleada y de la característica a partir de la cual se diferencien los grupos de población, la mayor parte de las investigaciones sobre la dimensión desigualdad de la segregación residencial usan de forma mayoritaria el Índice de Disimilaridad (D) o el de Segregación (S), siendo este último el más empleado en las investigaciones sobre la realidad española -Díaz, 2010; Parreño y Domínguez, 2008; Vono y Bayona, 2010-. En ambos casos, los grupos de población identificados en el interior del espacio urbano son tomados de dos en dos, mayoritario frente a uno de los minoritarios en el caso de $\mathrm{D}$, total de la población y uno de los subgrupos en los que ésta se divide, en el caso de $\mathrm{S}$.

Pero por su propia naturaleza estos índices no permiten estudiar la segregación multigrupo, esto es, aquella que considera la segregación existente entre los múltiples grupos de población definidos en la ciudad, en nuestro caso a partir de la nacionalidad. El instrumento estadístico más ampliamente utilizado para adentrarse en la diferenciación multigrupo es el Î́ndice de entropía de la segregación de Theil $(\mathrm{H})$ (Theil, 1972), que se basa en la medición de la entropía. Como es sabido, la entropía es una medida del desorden, y este índice puede definirse como el promedio ponderado de las diferencias de representación proporcional entre los grupos en las unidades espaciales empleadas (macroáreas, barrios, secciones censales, etc.) y la 
unidad mayor que las contiene (ciudad, en nuestro caso, municipio), ponderada por la población proporcional de las unidades espaciales (Linares, 2013). En nuestro caso, si la población de cada macroárea tuviese exactamente la misma composición por origen que la correspondiente al total municipal, o la población de cada barrio que conforma cada macroárea tuviese la misma composición por origen que la correspondiente al total de la macroárea, el índice tomaría un valor 0 . Y, por el contrario, una macroárea en la que los barrios no poseen diversidad - esto es, cada uno de ellos está habitado por un único grupo de población- se considera totalmente segregada, tomando el índice en este caso un valor de 1.

Su fórmula de cálculo es la siguiente:

$$
H=\sum_{i=1}^{k} \frac{t_{i}}{T}\left(\frac{E-E_{i}}{E}\right)
$$

Donde ti es la población total del área residencial i:

T es la población total de la ciudad;

Ei es la entropía del área residencial i, calculada de la siguiente manera:

$$
E_{i}=\sum_{r=1}^{n_{i}} Q_{r i} \ln \frac{1}{Q_{r i}}
$$

Donde Qri es la proporción del subgrupo de población $\mathrm{r}$ en la macroárea i, y n el número de subgrupos de población presentes en ella, $\mathrm{y}$

E la entropía de la ciudad, calculada de la siguiente manera:

$$
E=\sum_{r=1}^{n} Q_{r} \ln \frac{1}{Q_{r}}
$$

Donde Qr es la proporción del subgrupo de población $\mathrm{r}$ en el conjunto de la ciudad, y n el número de subgrupos de población presentes en ella.

Un elemento que debemos explicitar es que, siguiendo a Reardon et al. (2000), el índice puede ser calculado aunque alguno de los valores sea igual a cero, lo que resulta de fundamental importancia desde el momento en que, debido al nivel de desagregación espacial y temático de la variable empleada, estos casos son relativamente frecuentes, especialmente cuando nos referimos a los orígenes minoritarios de los extranjeros empadronados.

El uso de $\mathrm{H}$ es frecuente en las investigaciones sobre desigualdad de renta, pero lo es menos en aplicaciones a la segregación racial (Reardon et al., 2000) o residencial (Sánchez, 2012). En el caso de España, no son numerosas las aplicaciones a la segregación residencial, pudiéndose destacar entre ellas la de Madariaga et al. (2011), que emplean la renta como variable de diferenciación de la población. Sin embargo, 
tiene la ventaja de que su valor, que no está afectado por la magnitud de la población sobre la que se calcula, puede descomponerse en valores intragrupo o intergrupo, esto es, permite dar cuenta del aporte que hacen a la segregación total determinados niveles espaciales o determinados grupos de población. Esta última opción nos permitirá establecer qué parte de la segregación total del conjunto del municipio y de cada macroárea se debe a la separación entre españoles y el conjunto de extranjeros, y qué parte corresponde a la separación entre los seis grupos de extranjeros considerados.

Tabla 1. Equivalencia en entropía y potencial de redistribución para eliminar la desigualdad.

\begin{tabular}{|c|c|c|c|}
\hline Índice & \multicolumn{2}{|c|}{ Equivalencia en entropía } & Potencial de redistribución (\%) \\
\hline \multirow{2}{*}{0} & 50 & 50 & \multirow{2}{*}{$0 \%$} \\
\hline & 50 & 50 & \\
\hline \multirow{2}{*}{0,14} & 64 & 36 & \multirow{2}{*}{$14 \%$} \\
\hline & 36 & 64 & \\
\hline \multirow{2}{*}{0,29} & 70 & 30 & \multirow{2}{*}{$20 \%$} \\
\hline & 30 & 30 & \\
\hline \multirow{2}{*}{0,44} & 76 & 24 & \multirow{2}{*}{$26 \%$} \\
\hline & 24 & 76 & \\
\hline \multirow{2}{*}{0,5} & 78 & 22 & \multirow{2}{*}{$28 \%$} \\
\hline & 22 & 78 & \\
\hline \multirow{2}{*}{0,56} & 80 & 20 & \multirow{2}{*}{$30 \%$} \\
\hline & 20 & 80 & \\
\hline \multirow{2}{*}{0,75} & 87 & 13 & \multirow{2}{*}{$37 \%$} \\
\hline & 13 & 87 & \\
\hline
\end{tabular}

Fuente: Linares (2012).

Por último, debemos indicar que tiene bastante interés la denominada "equivalencia de entropía", una transformación que permite homologar el valor estandarizado de $\mathrm{H}$ con el correspondiente a una población dividida en dos clases (tabla 1); un valor de $\mathrm{H}$ de 0,44 implica que el 76\% del grupo A comparte el espacio con el $24 \%$ del grupo B, mientras que el $76 \%$ restante del grupo B sólo lo hace con el $24 \%$ del grupo A. El potencial de redistribución para obtener la máxima uniformidad es, en consecuencia, del $26 \%$, en otras palabras, el $26 \%$ de la población de cada grupo debería cambiar de residencia para obtener una distribución uniforme. 


\section{Los niveles de segregación en el municipio de Málaga}

\subsection{La segregación multigrupo}

En la tabla 2 se muestran los valores del índice de Theil multigrupo que, recordemos, considera simultáneamente la corresidencia de nuestros siete grupos de población. En ella puede observarse que el valor correspondiente a 2013 era de 0,3376, disminuyendo hasta situarse en 0,2991 en 2016, un descenso del -11,4\% (0,0385). Así pues, la desigualdad de la distribución de los grupos de población en el municipio de Málaga habría disminuido en los tres años de estudio.

Resulta complicado poner en contexto este resultado con otros correspondientes a la realidad española, ante la práctica ausencia de investigaciones que hayan empleado previamente este indicador atendiendo a la población discriminada según su nacionalidad. Sin embargo, acudiendo a la propiedad de equivalencia de entropía puede comprobarse cómo los valores en ambos años implican que el potencial de redistribución es bajo, por debajo del $25 \%$ en ambos casos (cfr. tabla 1). Así las cosas, tanto los valores reducidos de $\mathrm{H}$, como la tendencia a su descenso, están en la misma línea que las investigaciones a las que anteriormente hicimos referencia, que muestran una tendencia sostenida al descenso de los niveles de segregación desde 2003, empleando el índice de segregación, tanto para Málaga (Natera, 2012, Fernández, s.f) como para otras grandes ciudades españolas (Domínguez et al., 2010).

Tomadas individualmente, en 2013 la totalidad de las macroáreas presentaban valores del índice multigrupo menores a los del conjunto de Málaga (tabla 2), indicativos de unos niveles de segregación entre los siete grupos de población aún más bajos que los correspondientes al municipio. Así pues, la composición de la población de los barrios que están incluidos en cada una de las macroáreas era más parecida a la del conjunto de la macroárea que la distribución de la macroárea con respecto a la del total municipal; una situación, por tanto, más homogénea a gran escala que a pequeña escala. La lógica de esta realidad subyace en el hecho de que, como vimos, por cómo se construye la macroárea el espacio urbano de su interior resulta relativamente homogéneo. Una homogeneidad en cualquier caso mayor que la correspondiente a la comparación entre el total del municipio y las 19 macroáreas que lo componen, que se extienden por un tejido urbano tan dispar como el centro histórico, las ampliaciones de los años sesenta y setenta, o el tejido urbano poco denso de la periferia occidental. Todos son muy diferentes entre sí y presentan unas condiciones de vivienda, equipamientos y, por tanto, de precios, muy dispares. Reflejo de ello son los niveles de renta, que oscilaban entre los $17.009 € /$ hogar de la macroárea Palma/Palmilla y los 46.365 de Malagueta/Limonar en 2013 (Observatorio, 2015), o los precios de la vivienda, que en 2012 oscilaban entre los $2.891 € / \mathrm{m} 2$ de la Prolongación Alameda (incluida en la macroárea Mármoles) y los $1.703 € / \mathrm{m} 2$ de Churriana (Analistas, 2012). 
Tabla 2. Valores del Índice H de Theil multigrupo, y variación (2013/2016).

\begin{tabular}{|c|c|c|c|c|}
\hline Macroárea & 2013 & 2016 & Variación & $\%$ var. \\
\hline Total municipio & 0,3376 & 0,2991 & $-0,0385$ & $-11,40 \%$ \\
\hline Campanillas & 0,2493 & 0,2996 & $+0,0503$ & $+20,18 \%$ \\
\hline Centro & 0,3316 & 0,2845 & $-0,0471$ & $-14,20 \%$ \\
\hline Churriana/Bahía Málaga & 0,2376 & 0,1681 & $-0,0695$ & $-29,25 \%$ \\
\hline Ciudad Jardín & 0,3183 & 0,1144 & $-0,2039$ & $-64,06 \%$ \\
\hline El Palo/El Candado & 0,1016 & 0,0525 & $-0,0491$ & $-48,33 \%$ \\
\hline Huelin & 0,1965 & 0,2076 & $+0,0111$ & $+5,65 \%$ \\
\hline La Paz/P. Mediterráneo & 0,2164 & 0,1470 & $-0,0694$ & $-32,07 \%$ \\
\hline La Unión/C. Humilladero & 0,2246 & 0,2107 & $-0,0140$ & $-6,19 \%$ \\
\hline Luz/S. Andrés & 0,1162 & 0,1011 & $-0,0151$ & $-12,99 \%$ \\
\hline Malagueta/Limonar & 0,2448 & 0,1382 & $-0,1066$ & $-43,55 \%$ \\
\hline Mármoles/Carranque & 0,1239 & 0,1608 & $+0,0368$ & $+29,78 \%$ \\
\hline Palma/Palmilla & 0,0962 & 0,0814 & $-0,0148$ & $-15,38 \%$ \\
\hline Portada Alta/San Rafael & 0,1197 & 0,1730 & $+0,0533$ & $+44,53 \%$ \\
\hline Puerto de la Torre & 0,0827 & 0,1269 & $+0,0441$ & $+53,45 \%$ \\
\hline Puerta Blanca & 0,3043 & 0,4436 & $+0,1392$ & $+45,78 \%$ \\
\hline Segalerva/Olletas & 0,0631 & 0,0621 & $-0,0011$ & $-1,58 \%$ \\
\hline Suárez/Carlos Haya & 0,2469 & 0,1686 & $-0,0783$ & $-31,71 \%$ \\
\hline Teatinos & 0,0572 & 0,2106 & $+0,1534$ & $+268,18 \%$ \\
\hline Trinidad & 0,0947 & 0,0744 & $-0,0203$ & $-21,44 \%$ \\
\hline
\end{tabular}

Fuente: Padrones Municipales de Habitantes.Elaboración propia.

Tres años después, todas las macroáreas continuaban presentando valores del $\mathrm{H}$ multigrupo inferiores al del total municipal (tabla 2), con la excepción de Campanillas (con virtualmente el mismo valor que el total municipal) y Puerta Blanca. Pero aún en este último caso, el valor de $\mathrm{H}$ de 0,4436 implica un potencial de redistribución de la población de tan sólo el $26 \%$ (cfr. Tabla 1). 
Figura 2. Dinámicas de evolución de la población y evolución del valor del Índice de Theil por macroáreas (2013/2016).

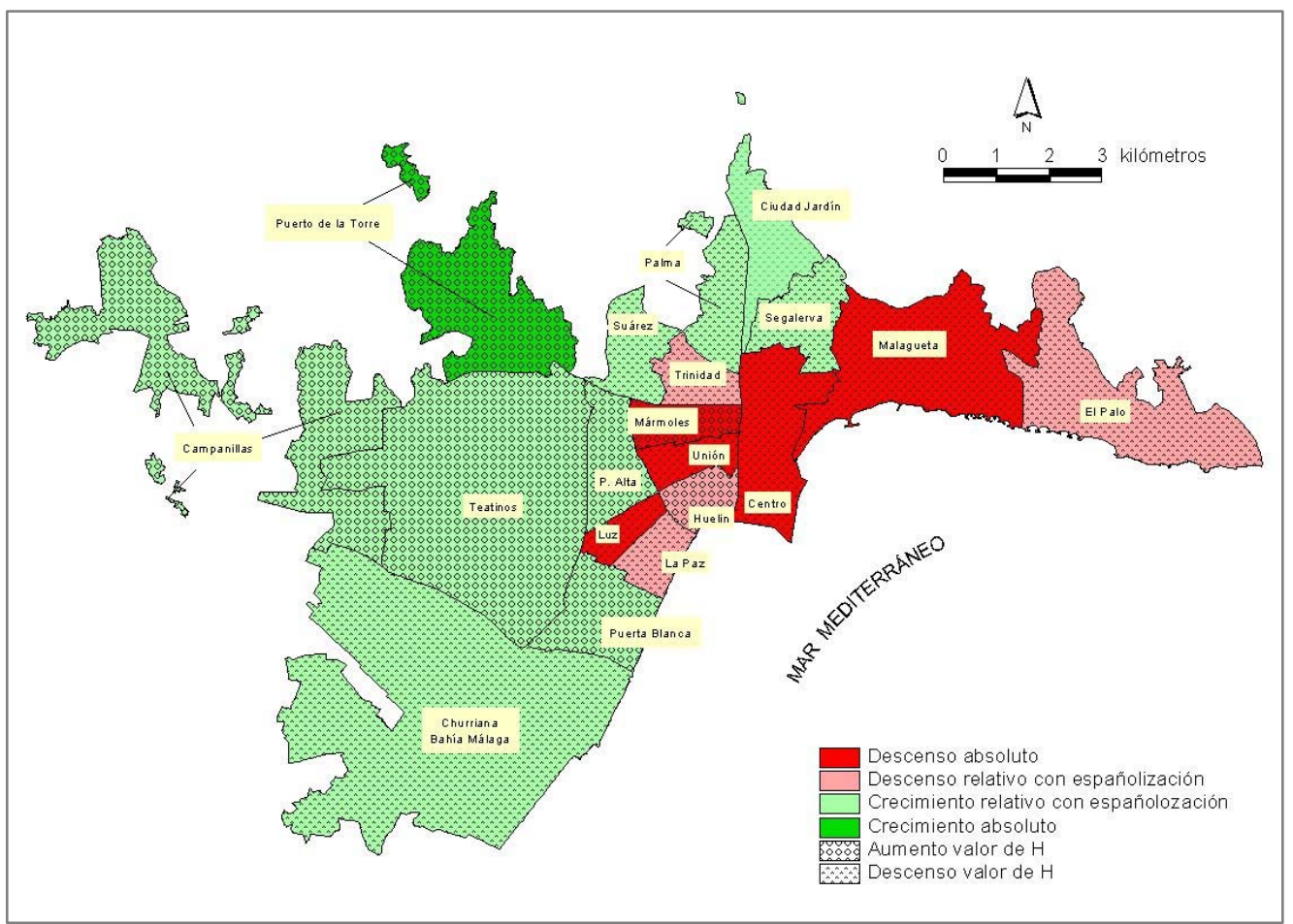

Fuente: Elaboración propia a partir de los datos padronales.

Sin embargo, la dinámica de evolución de los valores del índice en las macroáreas ha sido mucho más dispar. En un contexto de descenso global de la diferenciación multigrupo (valor municipal de $\mathrm{H}$ en descenso), 7 de las 19 han visto incrementarse el valor del índice (tabla 2), esto es, en ellas se han incrementado los niveles de desigualdad de la distribución de los grupos de población. De estas siete, cinco son asiento de alguna de las principales y más recientes actuaciones urbanísticas del municipio: Huelin y Puerta Blanca han experimentado importantes procesos de renovación urbana en sus frentes marítimos, en el marco de la consolidación de la expansión del Paseo Marítimo Antonio Banderas, en el litoral occidental de la ciudad. $\mathrm{Y}$, paralelamente, se han levantado grandes proyectos urbanísticos cristalizados en conjuntos residenciales cerrados en Teatinos (entre 2013 y 2015 se levantó aquí el $22,17 \%$ de todas las nuevas viviendas del municipio), al tiempo que se ha registrado una expansión del tejido urbano, más disperso, en Puerto de la Torre y Campanillas (dos de las únicas cinco macroáreas que vieron aumentar su densidad -calculada como habitantes/superficie urbanizada- en ese mismo periodo). Una dinámica expansiva que se refleja también en los niveles de crecimiento de la población de 
estas tres macroáreas; si el porcentaje de crecimiento del total de población municipal entre 2013 y 2016 fue del 0,12\% -698 personas-, el correspondiente a Teatinos fue del 8,78\% (3.655 nuevos empadronados), Campanillas del 5,29\% (907), y el Puerto de la Torre $2,07 \%$ (538 nuevos empadronados).

Tabla 3. Dinámica de la población por macroáreas y total municipal (2013/2016).

\begin{tabular}{|c|c|c|c|}
\hline Macroárea & Total población & Españoles & Extranjeros \\
\hline Total municipio & + & + & - \\
\hline Campanillas & + & + & - \\
\hline Centro & - & - & - \\
\hline Churriana/Bahía Málaga & + & + & - \\
\hline Ciudad Jardín & + & + & - \\
\hline El Palo/El Candado & - & + & - \\
\hline Huelin & - & + & - \\
\hline La Paz/P. Mediterráneo & - & + & - \\
\hline La Unión/C. Humilladero & - & - & - \\
\hline Luz/S. Andrés & - & - & - \\
\hline Malagueta/Limonar & - & - & - \\
\hline Mármoles/Carranque & - & - & - \\
\hline Palma/Palmilla & + & + & - \\
\hline Portada Alta/San Rafael & + & + & - \\
\hline Puerto de la Torre & + & + & + \\
\hline Puerta Blanca & + & + & - \\
\hline Segalerva/Olletas & + & + & - \\
\hline Suárez/Carlos Haya & + & + & - \\
\hline Teatinos & + & + & - \\
\hline Trinidad & - & + & - \\
\hline
\end{tabular}

Fuente: Padrones Municipales de Habitantes.Elaboración propia.

En su mayor parte las nuevas viviendas son libres, de calidad, y con unos precios comparativamente elevados, por lo que el acceso a la misma por parte de los inmigrantes laborales resulta más complicado que el del sector más solvente de los 
españoles. Aunque más adelante trataremos la diferenciación intergrupo, es pertinente indicar en este momento que el hecho de que haya aumentado el aporte de la separación entre españoles y total de extranjeros a la diferenciación total de estas macroáreas (tabla 4) refuerza el argumento de que el tipo de vivienda que se ha levantado en las macroáreas ha incidido sobre la evolución de los niveles de segregación de la población.

Estos hallazgos pueden ponerse en el contexto de la evolución del total de la población empadronada en Málaga, que es el resultado de la combinación de la evolución del número de españoles, por un lado, y del número de extranjeros, por otro. En la figura 2 mostramos esta información, condensada en cuatro dinámicas: crecimiento absoluto (tanto la población total, como la española y extranjeras aumentan entre 2013 y 2016); descenso absoluto (los tres contingentes disminuyen); crecimiento relativo con españolización (aumento de la población total y de la española, con descenso de los extranjeros); y descenso relativo con españolización (descenso del total de población y de los extranjeros, aumento de los españoles). Dinámicas que hemos combinado con la evolución (aumento o disminución) del valor de $\mathrm{H}$ entre 2013 y 2016. Así las cosas, en un contexto de aumento del total y de los españoles empadronados, y de descenso del volumen de extranjeros, como puede observarse en la tabla 3 la situación más común a nivel de macroárea es el descenso de este último grupo de población, con la única excepción del Puerto de la Torre. Este descenso es enjugado en 11 de ellas por el aumento de españoles, dando como resultado el incremento del total de población, al tiempo que tan sólo en las áreas centrales y pericentrales (Centro, La Unión, Luz, Mármoles y Malagueta) descienden todos los grupos. En las restantes 3 macroáreas el aumento de españoles no ha sido suficiente como para enjugar las pérdidas de población extranjera. En cualquier caso, la evolución de los contingentes es indicativa de la presencia de un proceso de sustitución de población extranjera por española.

Esta realidad supone un cambio de tendencia de la situación indicada por Natera y Batista (2012), que habían indicado que era la población extranjera la responsable del crecimiento de la población entre 2003 y 2010, no sólo a nivel municipal, sino en un número significativo de barrios de la ciudad. Además, en el 36,9\% de los barrios malagueños la población extranjera se había incrementado, al tiempo que la española disminuía, y todo ello en el contexto de llegadas masivas de inmigrantes laborales no sólo a Málaga, sino a su entorno más cercano, la Costa del Sol Occidental. Pero como acabamos de indicar la realidad actual es justamente la contraria, y cabe plantearse hasta qué punto este cambio de tendencia ha influido en los niveles de segregación de la población malagueña.

\subsection{La segregación intergrupo}

Como en el apartado de metodología habíamos indicado, una de las principales ventajas que tiene el índice de Theil sobre otros instrumentos estadísticos destinados a medir la desigualdad en el reparto de la población es que puede ser descompuesto en 
valores intergrupo e intragrupo. En nuestro caso es la primera descomposición la que nos interesa, puesto que nos permitirá comprobar si es la segregación entre españoles y total de extranjeros, o la segregación entre extranjeros, la que aporta más a la segregación total. Los resultados de la descomposición se muestran en la tabla 4 , y su distribución espacial en la figura 3.

Figura 3. Aportación de cada componente al valor total del Índice de Theil en 2016, por macroárea.

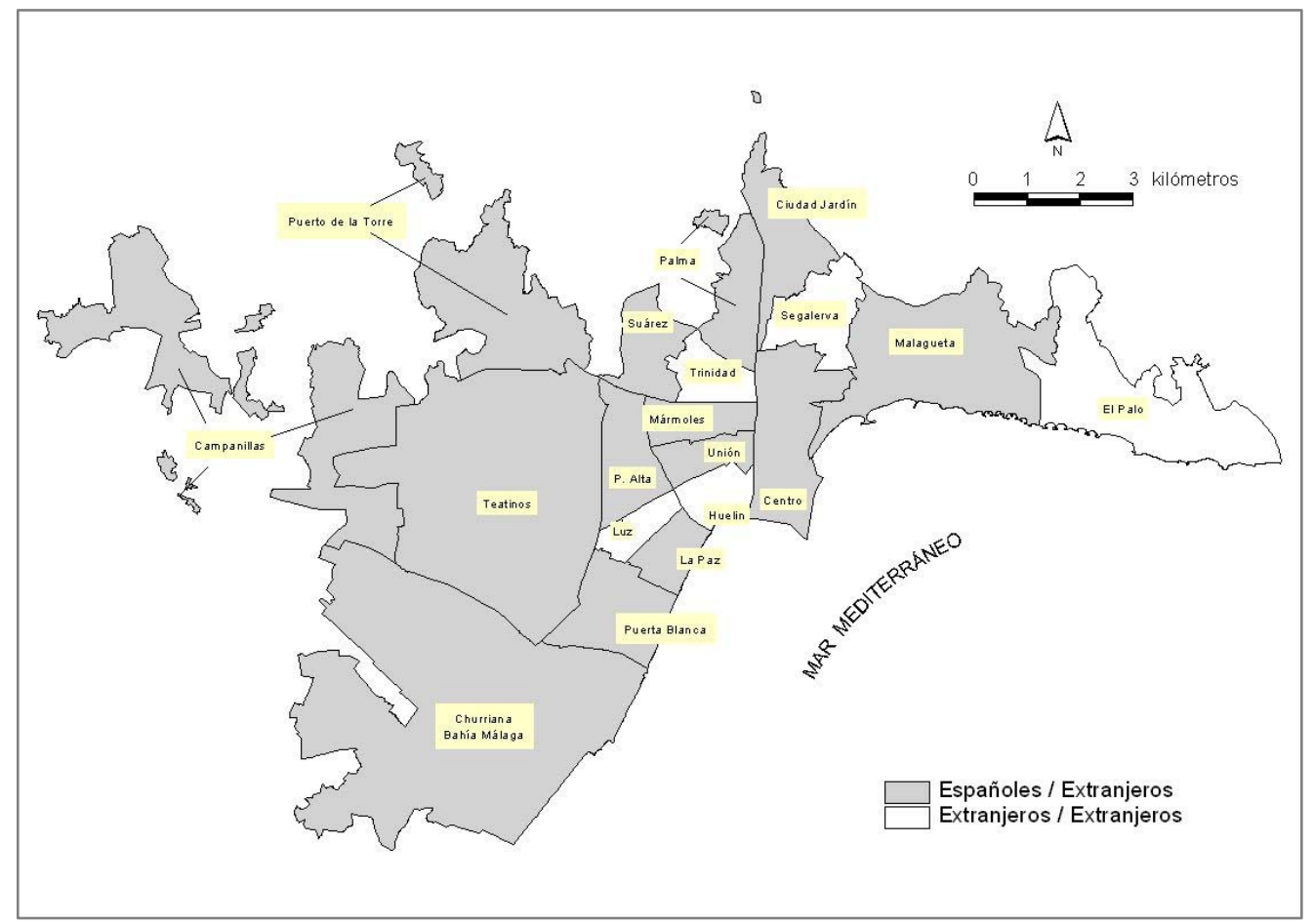

Fuente: Elaboración propia a partir de los datos padronales.

En ella puede observarse cómo cuatro quintas partes de la segregación total existente en el municipio se deben a la separación residencial de los españoles con respecto a los extranjeros, una distribución que se ha mantenido estable durante el periodo considerado. Los españoles, por tanto, tienden a separarse más en el espacio con respecto a los extranjeros que lo que lo hacen los extranjeros entre sí, siempre en un contexto, no lo olvidemos, de niveles de diferenciación residencial bajos (valores reducidos del $\mathrm{H}$ multigrupo). Como en su momento vimos, el grueso de los extranjeros residentes en el municipio malagueño tienen un marcado carácter laboral, por lo que su capacidad de elección de vivienda queda muy restringida a aquella más asequible económicamente (ya sea por compra o, básicamente, por alquiler). Es comprensible, por tanto, que los niveles de segregación entre extranjeros aporten poco 
a los niveles totales, puesto que el nicho residencial al que optan es básicamente el mismo y, en este contexto, las posibilidades de diferenciación espacial son escasas, máxime al nivel de municipio/macroárea.

Tabla 4. Descomposición del Índice H de Theil y aportación de cada componente (2013/2016).

\begin{tabular}{|c|c|c|c|c|c|c|}
\hline Macroárea & Padrón & H multigrupo & Esp./Extr. & $\%$ contribución & Extr./Extr. & $\%$ contribución \\
\hline \multirow{2}{*}{ Total municipio } & 2013 & 0,3376 & 0,2725 & $80,74 \%$ & 0,0650 & $19,26 \%$ \\
\hline & 2016 & 0,2991 & 0,2414 & $80,72 \%$ & 0,0577 & $19,28 \%$ \\
\hline \multirow{2}{*}{ Campanillas } & 2013 & 0,2493 & 0,2210 & $88,67 \%$ & 0,0282 & $11,33 \%$ \\
\hline & 2016 & 0,2996 & 0,2738 & $91,41 \%$ & 0,0257 & $8,59 \%$ \\
\hline \multirow{2}{*}{ Centro } & 2013 & 0,3316 & 0,2354 & $71,00 \%$ & 0,0962 & $29,00 \%$ \\
\hline & 2016 & 0,2845 & 0,2012 & $70,74 \%$ & 0,0832 & $29,26 \%$ \\
\hline \multirow[t]{2}{*}{ Churriana/ } & 2013 & 0,2376 & 0,1788 & $75,27 \%$ & 0,0587 & $24,73 \%$ \\
\hline & 2016 & 0,1681 & 0,1190 & $70,83 \%$ & 0,0490 & $29,17 \%$ \\
\hline \multirow{2}{*}{ Ciudad Jardín } & 2013 & 0,3183 & 0,2917 & $91,63 \%$ & 0,0266 & $8,37 \%$ \\
\hline & 2016 & 0,1144 & 0,0907 & $79,33 \%$ & 0,0236 & $20,67 \%$ \\
\hline \multirow{2}{*}{ El Palo/El Candado } & 2013 & 0,1016 & 0,0495 & $48,76 \%$ & 0,0520 & $51,24 \%$ \\
\hline & 2016 & 0,0525 & 0,0089 & $16,99 \%$ & 0,0436 & $83,01 \%$ \\
\hline \multirow{2}{*}{ Huelin } & 2013 & 0,1965 & 0,0242 & $12,32 \%$ & 0,1723 & $87,68 \%$ \\
\hline & 2016 & 0,2076 & 0,0518 & $24,95 \%$ & 0,1558 & $75,05 \%$ \\
\hline \multirow{2}{*}{ La Paz/P. Mediterráneo } & 2013 & 0,2164 & 0,1463 & $67,60 \%$ & 0,0701 & $32,40 \%$ \\
\hline & 2016 & 0,1470 & 0,0867 & $58,99 \%$ & 0,0603 & $41,01 \%$ \\
\hline \multirow{2}{*}{ La Unión/C. Humilladero } & 2013 & 0,2246 & 0,1444 & $64,28 \%$ & 0,0802 & $35,72 \%$ \\
\hline & 2016 & 0,2107 & 0,1339 & $63,55 \%$ & 0,0768 & $36,45 \%$ \\
\hline \multirow{2}{*}{$\begin{array}{c}\text { Luz/ } \\
\text { San Andrés }\end{array}$} & 2013 & 0,1162 & 0,0197 & $16,98 \%$ & 0,0965 & $83,02 \%$ \\
\hline & 2016 & 0,1011 & 0,0047 & $4,61 \%$ & 0,0965 & $95,39 \%$ \\
\hline \multirow{2}{*}{$\begin{array}{c}\text { Malagueta/ } \\
\text { Limonar }\end{array}$} & 2013 & 0,2448 & 0,1832 & $74,82 \%$ & 0,0616 & $25,18 \%$ \\
\hline & 2016 & 0,1382 & 0,0889 & $64,34 \%$ & 0,0493 & $35,66 \%$ \\
\hline Mármoles/ & 2013 & 0,1239 & 0,0666 & $53,77 \%$ & 0,0573 & $46,23 \%$ \\
\hline
\end{tabular}




\begin{tabular}{|c|c|c|c|c|c|c|}
\hline Carranque & 2016 & 0,1608 & 0,1087 & $67,64 \%$ & 0,0520 & $32,36 \%$ \\
\hline \multirow{2}{*}{ Puerto de la Torre } & 2013 & 0,0827 & 0,0515 & $62,20 \%$ & 0,0313 & $37,80 \%$ \\
\hline & 2016 & 0,1269 & 0,0939 & $74,02 \%$ & 0,0330 & $25,98 \%$ \\
\hline \multirow[t]{2}{*}{ Palma/ } & 2013 & 0,0962 & 0,0860 & $89,42 \%$ & 0,0102 & $10,58 \%$ \\
\hline & 2016 & 0,0814 & 0,0731 & $89,74 \%$ & 0,0084 & $10,26 \%$ \\
\hline Portada Alta/ & 2013 & 0,1197 & 0,0735 & $61,40 \%$ & 0,0462 & $38,60 \%$ \\
\hline San Rafael & 2016 & 0,1730 & 0,1328 & $76,78 \%$ & 0,0402 & $23,22 \%$ \\
\hline \multirow{2}{*}{ Puerta Blanca } & 2013 & 0,3043 & 0,2671 & $87,76 \%$ & 0,0373 & $12,24 \%$ \\
\hline & 2016 & 0,4436 & 0,4125 & $93,00 \%$ & 0,0310 & $7,00 \%$ \\
\hline \multirow[t]{2}{*}{ Segalerva/ } & 2013 & 0,0631 & 0,0119 & $18,87 \%$ & 0,0512 & $81,13 \%$ \\
\hline & 2016 & 0,0621 & 0,0142 & $22,82 \%$ & 0,0479 & $77,18 \%$ \\
\hline \multirow{2}{*}{ Suárez/Carlos Haya } & 2013 & 0,2469 & 0,1855 & $75,12 \%$ & 0,0614 & $24,88 \%$ \\
\hline & 2016 & 0,1686 & 0,1187 & $70,38 \%$ & 0,0499 & $29,62 \%$ \\
\hline \multirow{2}{*}{ Teatinos } & 2013 & 0,0572 & 0,0246 & $42,94 \%$ & 0,0326 & $57,06 \%$ \\
\hline & 2016 & 0,2106 & 0,1856 & $88,16 \%$ & 0,0249 & $11,84 \%$ \\
\hline \multirow{2}{*}{ Trinidad } & 2013 & 0,0947 & 0,0225 & $23,78 \%$ & 0,0721 & $76,22 \%$ \\
\hline & 2016 & 0,0941 & 0,0220 & $23,37 \%$ & 0,0721 & $76,63 \%$ \\
\hline
\end{tabular}

Fuente: Padrones Municipales de Habitantes.Elaboración propia.

En la tabla 4 también se aprecia cómo la mayor parte de las macroáreas comparten la situación que el conjunto del municipio: en ellas la porción mayoritaria de la diferenciación se debe a las diferencias residenciales de los españoles con respecto al conjunto de extranjeros, en ambos años estudiados. Sin embargo, hay un grupo en las que la mayor parte de la diferenciación residencial presente se debe a las diferencias residenciales entre los grupos de extranjeros: en 2013, Huelin, Luz-San Andrés, Segalerva-Olletas, Trinidad, Teatinos y El Palo-El Candado. En 2016 la nómina se repite con la única excepción de Teatinos, donde es el componente españoles/extranjeros el que aporta el grueso a la diferenciación total.

Podemos ejemplificar estos casos con los correspondientes a tres macroáreas: El Palo/El Candado, Trinidad y Segalerva/Olletas. Las tres tienen en común el hecho de que los niveles de diferenciación entre grupos de población son muy bajos, con unos potenciales de redistribución de la población por debajo del 10\% en los dos años (cfr. tablas 4 y 1). Además, en todas ellas está presente el proceso de sustitución de población extranjera por española al que anteriormente hicimos referencia, con 
pérdidas absolutas de población excepto en Segalerva, donde, de todas formas, el aumento de población ha sido testimonial -54 personas, el $0,23 \%$ sobre el total de empadronados en 2013-.

Como puede observarse en la tabla 4, en la primera de ellas, El Palo/El Candado, se ha registrado un descenso del valor de $\mathrm{H}$ multigrupo a la mitad, derivado de un desplome de la diferenciación entre españoles y total de extranjeros: la población de éstos últimos ha descendido en un $20 \%$, aumentando la concentración relativa de los restantes en el barrio de El Palo, el más poblado de la macroárea, y reduciéndose en los demás. Por tanto, las diferencias residenciales entre españoles y extranjeros han disminuido, al concentrarse ambos grupos de forma muy mayoritaria en uno solo de los barrios, que concentra por sí mismo el $38 \%$ de la población de la macroárea. Y aunque también ha disminuido el valor de la diferenciación de los extranjeros entre sí, lo ha hecho en mucha menor medida que la anterior; en consecuencia, en términos porcentuales el aporte de las diferencias residenciales entre extranjeros a la diferenciación total ha pasado de un $51 \%$ en 2013 a un $86 \%$ en 2016. De cualquier manera, el hecho de que en ambos años sea la diferenciación entre extranjeros la principal responsable de la diferenciación total de la macroárea encuentra su explicación en los grandes contrastes existentes en su trama urbana. Estos contrastes quedan claramente marcados por Playas de El Palo (el único barrio identificado como vulnerable 2011 en toda el área oriental del municipio por el Ministerio de Fomento 2015-), y El Candado o Pinares de San Antón, asiento de viviendas y urbanizaciones con muy buenas calidades residenciales. Los contrastes residenciales tienen su reflejo en el origen mayoritario de los extranjeros empadronados en ellos: en Playas del Palo el $67 \%$ de los extranjeros empadronados eran latinoamericanos, magrebíes o europeos del este, mientras que en los otros dos el $56 \%$ de los extranjeros procedían de países desarrollados, diferencias que son las que ha reflejado el índice.

También en Segalerva-Olletas se aprecia el proceso de sustitución de población extranjera por española, solo que en esta ocasión el aumento de españoles ha sido superior a las pérdidas de los extranjeros, dando lugar a un aumento del total de población. En esta macroárea merece la pena destacar el hecho de que la presencia en los barrios que la conforman de latinoamericanos y magrebíes es disímil. A modo de ejemplo la población marroquí triplica la latinoamericana, mientras que en Conde de Ureña la situación es justamente la contraria. Estas diferencias ya habían sido puestas de manifiesto por Natera (2012), quien había mostrado, empleando cartografía del indicador de autocorrelación espacial local (LISA), cómo concentraciones estadísticamente significativas alto-alto de los magrebíes se correspondían con concentraciones bajo-bajo de latinoamericanos. Además, Thayer (2007) ya indicó la existencia de una voluntad por parte de los ecuatorianos residentes en Madrid de separarse físicamente del colectivo marroquí, que estaría sustentada, en parte, por un prejuicio previo de los ecuatorianos para con ellos.

Por último, en la macroárea Trinidad en 2016 el porcentaje de extranjeros era elevado en relación al del total municipal (11,97\% frente a 7,74\%, respectivamente), aun contando con la pérdida de volumen experimentada por este grupo de población 
desde 2013 (505 empadronados). Una evolución que contrasta con la correspondiente a los españoles, que han visto incrementarse su número. Se trata, en cualquier caso, de población con bajos niveles de instrucción, y elevados niveles de paro (Martín, 2015), por lo que sus posibilidades de obtener vivienda, su fuerza en el mercado inmobiliario, no es muy diferente a la de los extranjeros laborales con los que comparten el espacio, y de ahí el escaso aporte a la diferenciación total del binomio españoles/extranjeros, en un tejido urbano escasamente renovado. Por otro lado, el comienzo de la inmigración en la zona arrancó en los ochenta, con un especial protagonismo de los marroquíes, que vieron multiplicado su número gracias a la aparición de redes de apoyo, y por el incremento de las reagrupaciones familiares, lo que ha acabado fomentando una localización espacial relativamente concentrada de este colectivo. Y, en los últimos años, ha tenido un protagonismo especial la radicación de europeos del Este, fundamentalmente ucranianos, y latinoamericanos, introduciendo elementos de diferenciación espacial que estarían en la base del mayor peso de la separación entre grupos de extranjeros en el total de la segregación de la macroárea.

\section{Conclusiones}

El municipio de Málaga ha venido experimentando desde 2013 un descenso en el número de extranjeros empadronados, revertiendo una dinámica de crecimiento que se mantenía continua desde comienzos de siglo. Y aunque su distribución espacial no ha sido, ni es, homogénea, los niveles de diferenciación residencial hasta ese año eran bajos, y en descenso. Cómo ha influido sobre estos niveles el descenso de extranjeros era uno de los objetivos de los párrafos anteriores, pero el interés de los mismos reside no tanto en la constatación efectiva de que los niveles de diferenciación residencial en el municipio de Málaga continúan siendo bajos, y todavía en descenso, sino en las posibilidades que ofrece el instrumento estadístico que hemos empleado: el Índice de Theil $(\mathrm{H})$, que mide la diferenciación residencial multigrupo, y cuya descomposición intergrupo permite enriquecer notablemente la interpretación de la diferenciación residencial en nuestras ciudades.

En este sentido, dicha descomposición ha señalado la existencia de macroáreas en las que la mayor parte de la diferenciación residencial se debe a la separación entre extranjeros. Hemos identificado varias situaciones que darían explicación a este hecho. Una, correspondiente a El Palo/El Candado, en la que son las propias diferencias en las características tipológicas del tejido urbano las que están en la base de este hecho. Otra, en la que la separación "sociológica" entre grupos de extranjeros (latinoamericanos y magrebíes) podría argumentarse como explicación parcial a la situación de Segalerva/Olletas, un hecho que está en consonancia con hallazgos referidos al propio municipio y a la Comunidad de Madrid, a la que habría que unir a los residentes provenientes de Europa del Este en la macroárea Trinidad.

Además, también hemos obtenido evidencias de que el reciente proceso de aparición de tejido urbano compuesto por vivienda libre, de calidad, en determinadas 
macroáreas ha tenido su reflejo no tanto en los niveles de diferenciación residencial de las mismas, que se mantiene bajo, sino en la composición de la diferenciación. En las cinco macroáreas que han experimentado este proceso se ha incrementado la separación residencial entre españoles y total de extranjeros, un hecho que no podría haber quedado registrado si hubiésemos empleado los instrumentos clásicos de medición de la diferenciación residencial.

Pareciera, por tanto, que el empleo de este índice puede ser útil a la hora de profundizar en las características, y dar pistas sobre las causas, de la diferenciación residencial de la población. No obstante, creemos que es importante señalar que un aspecto problemático en lo relativo a la aplicación de índices relativamente complejos, como el que nos ocupa, es el correspondiente a la unidad espacial de referencia. Nosotros hemos empleado macroáreas construidas a partir de la agregación de barrios. Dado que los límites de éstos últimos no varían una vez establecidos, los problemas derivados del MAUP están ausentes en el estudio de la evolución temporal de la variable, lo que no ocurriría si se empleasen las secciones censales. Pero además, no debemos perder de vista el hecho de que estamos tratando con la distribución de la población en el espacio urbano, una distribución en la que en gran medida subyacen condicionantes no sólo económicos, sino sociológicos. Por ello tiene sentido emplear una unidad espacial de referencia con algún sentido sociológico, una unidad espacial con la que el habitante se sienta identificado, y que sea lo más homogénea posible en lo relativo a las características de su tejido urbano. La sección urbana, por su propia naturaleza, no cumple en la mayor parte de las ocasiones con estas condiciones, la población no se identifica con la sección en la que está encuadrada, y ésta puede abarcar porciones de la ciudad muy disímiles entre sí -uniendo, por ejemplo, polígonos industriales con bloques de vivienda, especialmente en las periferias urbanas-.

Por otro lado, podemos plantearnos también si los valores de $\mathrm{H}$ apuntarán a unos niveles de segregación, y a unas contribuciones al valor total por parte de los grupos implicados, diferentes a las que hemos obtenido si empleásemos unidades espaciales más pequeñas. Esta es una cuestión de indudable interés, pero que tan sólo puede abordarse si se cuenta con información referida a unidades espaciales menores que el barrio -o la sección, en su caso-. Estas unidades podrían ser las manzanas, caso en el que el binomio sería manzana/barrio, en lugar del barrio/macroárea que nosotros hemos empleado. Sin embargo, el acceso a la información necesaria a este nivel de desagregación espacial no está asegurado, básicamente por razones de secreto estadístico, como tampoco lo está si acudimos a las grids más al uso, para las cuales la disponibilidad real de información es totalmente insuficiente para un estudio como el que hemos realizado. Es una cuestión pendiente, que podrá ser abordada en paralelo a la disponibilidad de información relevante a estos niveles de desagregación espacial.

En este sentido, somos conscientes de que no todas nuestras ciudades cuentan con una delimitación espacial alternativa a la sección censal que contenga información demográfica útil, y referida a diferentes momentos temporales. Mientras esta 
situación se mantenga la aplicación de los instrumentos de medida de la diferenciación residencial deberá continuar quedando referida a unidades espaciales con límites cambiantes y que fueron construidas con necesidades diferentes a la de los investigadores sociales en mente, limitando en cierta medida la interpretación de los resultados obtenidos.

\section{Bibliografía}

Achebak, H., y Bayona-i-Carrasco, J (2015): Asentamiento territorial y diversidad de la población marroquí en España. En García Castaño, F.J., Megías Megías, A., y Ortega Torres, J. (Eds.): Actas del VIII Congreso sobre Migraciones Internacionales en España. Granada, 16/18/9/2015. pp.S07/03-S07/16.

http://butlleticed.uab.es/butCED/num97_fitxers/Migrac.Granada_S07_Achebak_Bayona.pdf Analistas Económicos de Andalucía (2012): Evolución del mercado inmobiliario en Málaga y perspectivas de futuro. Málaga, Fundación CIEDES.

ciedes.es/images/stories/Cuadernos/Cuaderno13.pdf

Batista Zamora, A.E., y Natera Rivas, J.J. (2013): Extranjeros en la Costa del Sol Occidental: diferenciación residencial y caracterización de su distribución espacial. Cuadernos Geográficos de la Universidad de Granada, 52, 264-287.

http://revistaseug.ugr.es/index.php/cuadgeo/article/view/934/1085

Bonillo, D., Belmonte, L.J., y Román, I. (2011): Estudio socioeconómico de las condiciones de vida de la población extranjera en España: el caso particular de Almería. European Journal of Psychology of Education, 4(2), 87-107.

https://formacionasunivep.com/ejep/index.php/journal/article/download/70/95

Checa Olmos, J.C., Arjona Garrido, A., y Checa Olmos, F. (2011): Segregación residencial de la población extranjera en Andalucía, España. Papeles de Población, 17 (70), 219-246.

Cutillas Orgilés, E. (2009): Jubilados europeos en la provincia de Alicante. La segregación residencial en la ocupación del territorio. En López Trigal, L., Abellán, A., y Goudenau, D. (Coords.): Envejecimiento, población y territorio. Actas de la XI Reunión del Grupo de Población de la Asociación de Geógrafos Españoles. León, Asociación de Geógrafos Españoles, 441-454. http://hdl.handle.net/10045/16461

Díaz Parra, I. (2010): Distribución y segregación de la población residente extranjera en el municipio de Sevilla (2002-2008). Estudios Geográficos, 71 (268), 130-128.

http://dx.doi.org/10.3989/estgeogr.0470

Domíngez Mújica, J., Parreño Castellano, J.M., y Díaz Hernández, R. (2010): Inmigración y ciudad en España. Integración versus segregación socio-territoriales. Scripta Nova. Revista Electrónica de Geografía y Ciencias Sociales, XIV (331). http://www.ub.es/geocrit/sn/sn331/sn-331-50.htm

Echezarra, A. (2010): Segregación residencial de los extranjeros en el área metropolitana de

Madrid. Un análisis cuantitativo. Revista Internacional de Sociología, 68 (1), 165-197. http://dx.doi.org/10.3989/ris.2007.11.12 
Egea Jiménez, C., Nieto Calmaestra, J.A., Domínguez Clemente, J., y González Rego, R.A. (2008): Vulnerabilidad del tejido social de los barrios desfavorecidos. Análisis y potencialidades. Sevilla, Centro de Estudios Andaluces.

https://www.centrodeestudiosandaluces.es/datos/factoriaideas/ifo11_08.pdf

Fernández Morales, Antonio (s.f.): Desigualdad, pobreza, exclusión social y segregación residencial en Málaga. Málaga. Ayuntamiento de Málaga/Observatorio del Medio Ambiente Urbano. http://www.omau-malaga.com/agenda21/subidas/archivos/arc_116.pdf

Galeano, Juan, y Bayona i Carrasco, Jordi (2016): Diversidad poblacional e inmigración en España: un análisis de la evolución metropolitana reciente. En Domínguez Mújica, J., y Díaz Hernández, R. (Coords.): Actas XV Congreso de la población española. Población y territorio en la encrucijada de las ciencias sociales. Servicio de Publicaciones de la Universidad de Las Palmas de Gran Canaria, 199-213.

Huete García, M.A., y y Muñoz Moreno, R. (2011): Modelos de barrio y lógicas de localizacón de la población inmigrante. El caso de la ciudad de Sevilla. Scripta Nova. Revista electrónica de Geografía y Ciencias Sociales. XV (372).

http://www.ub.es/geocrit/sn/sn-372.htm

Huete García, M.A., y Mantecón, A. (2011): Más allá del turismo: movilidad residencial y nuevos núcleos urbanos. Boletín de la Asociación de Geógrafos Españoles, 56. pp.111-128. http://www.age-geografia.es/ojs/index.php/bage/article/view/1346/1269

Linares, S. (2012): Análisis y modelización de la segregación socioespacial en ciudades medias bonaerenses mediante Sistemas de Información Geográfica: Pergamino, Olavarría y Tandil (1991-2001). Revista Geográfica de Valparaíso, 45, 3-22.

http://geografiapucv.cl/wp-content/uploads/2016/05/45_1.pdf

Linares, S. (2013): Medidas de segregación socioespacial: discusión metodológica y aplicación empírica sobre ciudades medias argentinas. Persona y Sociedad, 27(2), 11-40.

http://www.personaysociedad.cl/ojs/index.php/pys/article/view/88

Madariaga, R., Martori, J.C., Oller, R. (2011): Distribución espacial y desigualdad de la renta salarial en el área metropolitana de Barcelona. Scripta Nova. Revista electrónica de Geografía y Ciencias Sociales, XVI (405). http://www.ub.edu/geocrit/sn/sn-405.htm

Mantecón, A., Huete, R,, y Mazón, T. (2009): Las urbanizaciones "europeas". Una investigación sobre las nuevas sociedades duales en el Mediterráneo. Scripta Nova Revista electrónica de Geografía y Ciencias Sociales, XIII (301).

http://www.ub.es/geocrit/sn/sn-301.htm

Martín Cots, P. -dir.- (2015): Estrategia urbana integrada sostenible "Perchel Lagunillas". Málaga, OMAU, Ayuntamiento de Málaga

http://static.omau-malaga.com/omau/subidas/archivos/2/1/arc_7712.pdf

Martínez del Olmo, A., y Leal Maldonado, J. (2008): La segregación residencial, un indicador espacial confuso en la representación de la problemática residencial de los inmigrantes económicos: el caso de la comunidad de Madrid. Arquitectura, ciudad y entorno, III (8), 53-64.

Martínez Martín, R. (2011): Los trabajadores inmigrantes en Andalucía: trayectorias laborales e integración social. Temas Laborales, 108, 145-171. 
https://www.juntadeandalucia.es/empleo/carl/carlportal-

portlets/documentos?nombre=33_1240_3.pdf

Martínez Martín, R., y Prior Ruiz, J. C. (2001): La inserción socioprofesional de los inmigrantes en Andalucía. Papers, 96 (3), 883-911.

Ministerio de Fomento (2015): Atlas de la vulnerabilidad urbana 2011. Aplicación web accesible en http://atlasvulnerabilidadurbana.fomento.es/

Natera Rivas, J.J. (2012): "Población extranjera en el municipio de Málaga. Evolución de sus niveles de diferenciación residencial y de sus pautas de distribución espacial (2003/2010). Scripta Nova. Revista Electrónica de Geografía y Ciencias Sociales. [En línea]. Barcelona: Universidad de Barcelona, 10 de septiembre de 2012, vol. XVI, nº 413.

http://www.ub.es/geocrit/sn/sn-413.htm.

Natera Rivas, J.J., y Batista Zamora, A.E. (2012): Influencia de la población extranjera en la evolución y estructura por edad de la población del municipio de Málaga. Anales de Geografía de la Universidad Complutense. 32, 323-344.

http://revistas.ucm.es/index.php/AGUC/article/view/39723/38215

Ocaña Ocaña, C. (2005a). Microanálisis sociodemográfico de espacios urbanos. Boletín de la AGE, 40, 5-34. http://www.age-geografia.es/ojs/index.php/bage/article/view/2007/1920

Ocaña Ocaña, C. (2005b). La inmigración extranjera en el mapa social de la ciudad de Málaga. Baetica, 27, 217-232. http://hdl.handle.net/10630/6890

Observatorio del Medioambiente Urbano (2015): Cohesión social y desarrollo económico. Agenda Urbana 2015. Málaga, Ayuntamiento de Málaga/Observatorio del Medio Ambiente Urbano. http://www.omau-malaga.com/agenda21/subidas/archivos/arc_216.pdf

Openshaw, S. (1984): The modifiable areal unit problem. Norwich, Geobooks. http://www.qmrg.org.uk/catmog/index.html\#

Ortega Rivera, E. (2012): Análisis demográfico del empadronamiento: la población de nacionalidad extranjera en Andalucía (1998-2010). Papers de Geografía, 402, 66 págs.

Parreño Castellano, Juan Manuel, y Domínguez Mújica, Josefina (2008): Extranjería y diferenciación residencial en Canarias: la perspectiva del microanálisis espacial. Investigaciones Geográficas, 45, 163-199. http://dx.doi.org/10.14198/INGEO2008.45.07

Reardon, S.F., Yun, J.T, y Mcnulty Eitule, T. (2000): The changing structure of school segregation: measurement and evidence of multirracial metropolitan-area school segregation, 1989-1995. Demography, 37 (3), 351-364.

http://www.jstor.org/stable/2648047

Rodríguez Vignoli, J. (2001): Segregación residencial socioeconómica: ¿qué es?, ¿cómo se mide?, ¿qué está pasando?, ¿importa? Santiago de Chile CEPAL.

http://repositorio.cepal.org/handle/11362/7149

Sánchez Peña, L. (2012): ¿Viviendo cada vez más separados? Un análisis multigrupo de la segregación residencial en la ciudad de México, 1990-2005. Estudios demográficos y urbanos, 27 (1), 57-93.

http://estudiosdemograficosyurbanos.colmex.mx/index.php/edu/article/view/1405/1398

Thayer Correa, L.E. (2007): Inmigrantes ecuatorianos en la Comunidad de Madrid. La apropiación del espacio y la expropiación del tiempo. Madrid, Editorial Complutense. www.flacsoandes.edu.ec/web/imagesFTP/6648.THAYER.pdf 
Theil, H. (1972): Statistical decomposition analysis. With applications in the Social -and Administrative Sciences. Amsterdam, North-Holland Publishing Company.

Vono, G., y Bayona i Carrasco, J. (2010): El asentamiento residencial de los latinoamericanos en las principales ciudades españolas (2001-2009). Notas de Población, 91, 129-159.

http://repositorio.cepal.org/bitstream/handle/11362/12875/np91129159_es.pdf?sequence=1\&is Allowed $=\mathrm{y}$ 